\title{
EVALUATION OF IMMEDIATE AND LATE COMPLICATIONS IN BREAST RECONSTRUCTION WITH IMPLANTS IN PATIENTS WITH BREAST CANCER TREATED IN A TERTIARY SERVICE IN CEARÁ
}

Aline Carvalho Rocha', Lays Samara da Costa Silva e Silva', Gina Zully Carhuancho Flores', Cristina Poliana Rolim Saraiva dos Santos' ${ }^{1}$ Gabriela Carvalho Pinheiro ${ }^{1}$

${ }^{1}$ Maternidade Escola Assis de Chateaubriand - Fortaleza (CE), Brazil

Introduction: The technique of breast reconstruction using silicone implants is the predominant way for breast reconstruction worldwide. Over the last years there has been a growing interest in performing immediate reconstruction with single stage implantation after mastectomy as an attempt to simplify the reconstruction procedure and improve psychosocial morbidity, as well reducing costs. The reconstruction with single stage suggests a slightly higher complication rate related to implant loss. Objectives: To evaluate the immediate complications (up to 30 days after surgery) and late complications (30 days to two years after surgery) resulting from immediate breast reconstruction with implants in patients with breast cancer treated at a tertiary hospital in Ceará. Methods: Analytical observational study of a retrospective cohort, from the review of medical records of patients undergoing immediate breast reconstruction with prosthesis or temporary extensor after mastectomy for breast cancer, at Maternidade Escola Assis de Chateaubriand, from 2015 to 2019. Establishing the prevalence among the common characteristics related both to the surgical procedure to which they were submitted, and to the clinical-epidemiological profile of the patients. Results: The study was made with 63 women who underwent immediate breast reconstruction with a mean age of 47 years. It was observed that immediate and onetime breast reconstruction was the most prevalent, contributing with $77.7 \%$ of the cases analyzed. In 19 cases (30.1\%), we observed some type of surgical complication, with surgical wound dehiscence being the most frequent (42.1\%). Other complications detected were infection and implant loss (21.1\%), capsular contracture (15.8\%), seroma (10\%), cellulitis (5.3\%), and rupture of the expander (5.3\%). Surgical complications were assessed according to the type of implant (prosthesis or temporary expander), use of radiotherapy and clinical conditions of patients. No statistical significance was found for these variables with surgical complications. Only the type of mastectomy performed was statistically significant, with radical mastectomy associated with half of the complications $(\mathrm{p}=0.045)$. Conclusions: Immediate breast reconstruction with single-time implant was the most used technique in our service, with a postoperative complication rate of around $30 \%$. However, the biggest complication was the dehiscence of the surgical wound, but the infection of the implant did not cause the loss of reconstruction. Radiotherapy was not related to complications until two years after surgery. 